\title{
Relation between object properties and EMG during reaching to grasp
}

\author{
Nadine Fligge*, Holger Urbanek, Patrick van der Smagt \\ German Aerospace Center (DLR), Center for Robotics and Mechatronics, Muenchner Strasse 20, D-82234 Oberpfaffenhofen-Wessling, Germany
}

\section{A R T I C L E I N F O}

\section{Article history:}

Received 23 March 2012

Received in revised form 6 August 2012

Accepted 22 October 2012

\section{Keywords:}

Object size

sEMG

Reaching movement

\begin{abstract}
A B S T R A C T
In order to stably grasp an object with an artificial hand, a priori knowledge of the object's properties is a major advantage, especially to ensure subsequent manipulation of the object held by the hand. This is also true for hand prostheses: pre-shaping of the hand while approaching the object, similar to able-bodied, allows the wearer for a much faster and more intuitive way of handling and grasping an object. For hand prostheses, it would be advantageous to obtain this information about object properties from a surface electromyography (sEMG) signal, which is already present and used to control the active prosthetic hand.

We describe experiments in which human subjects grasp different objects at different positions while their muscular activity is recorded through eight sEMG electrodes placed on the forearm. Results show that sEMG data, gathered before the hand is in contact with the object, can be used to obtain relevant information on object properties such as size and weight.
\end{abstract}

(c) 2012 Elsevier Ltd. All rights reserved.

\section{Introduction}

Human reaching and grasping is a complex task and a vast body of research is devoted to it. One important reason for this research is the fact that it can be very applicable to robotic hand/arm systems, as well as it will be useful for the development of more dexterous active hand prostheses. A central problem in robotic and prosthetic grasping is to obtain information on the object that has to be grasped: its shape, weight, and intended use are all factors determining the position and exerted force of the fingers grasping the object; see, e.g., Cutkosky and Howe (1990) for an overview of human grasping studies and challenges in grasping with artificial hands. Even though research on the control of active hand prosthesis controlled with sEMG signals made considerable progress recently (see, e.g., Parker et al. (2006) for an overview), this research mainly concentrates on recognizing different finger movements.

sEMG-based control of active hand prosthesis can be realized in two different fashions. The first is to attempt to restore single finger (or single joint) movement. First of all this requires highly advanced sEMG methods, which can distinguish the movement of each finger in each of its movement directions (including abduction/adduction) from a mixed sEMG signal, but also asks for a prosthetic hand which is able to move each of the fingers correspondingly-a requirement which is at best partly given by current marketed prosthetic hands. A second approach, which is therefore

\footnotetext{
* Corresponding author.

E-mail addresses: nadine.fligge@dlr.de (N. Fligge), Holger.Urbanek@dlr.de (H. Urbanek), Smagt@dlr.de (P. van der Smagt).
}

more practiced, is to extract the intended grasp from an sEMG signal and shape the hand accordingly.

When more complex grasps are required, e.g., distinguishing between picking up a pen to write with, a cup to drink from, or a plate to carry, a better sEMG interface is required. In order to optimize a grasp quickly and intuitively, knowledge on the shape and approximate weight of the object is essential, allowing the prosthetic hand to be pre-shaped w.r.t. aperture and finger stiffness.

Previous research has shown the ability to detect single finger movement from sEMG (Bitzer and van der Smagt, 2006) as well as finger force (Castellini and van der Smagt, 2009); but only as soon as the object is being held, therefore such approaches cannot be used for pre-planning the grasp.

It has been previously demonstrated that, during the reaching movement, hand aperture increases to a maximum which is related linearly to the object's size, and this maximum aperture is reached about midway in the reaching movement (e.g., Jeannerod, 1981; Paulignan and Jeannerod, 1996; Santello and Soechting, 1998). Furthermore, it has been shown that human hand pre-shaping (Santello and Soechting, 1998) and hand configuration (Schettino et al., 2003) during a reaching movement depend on object size and shape, and that this pre-shaping is initiated in the first half of the reaching movement. Eastough and Edwards (2007) showed an influence of object weight on the grasp kinematics during the reaching movement.

Clearly, there is information on the object properties in the movement of the fingers during reach movements. We investigate the usability of this information from forearm sEMG signals.

Brochier et al. (2004) were able to distinguish between different objects by using EMG-signals recorded from electrodes implanted 
in the hand, arm, and finger muscles of monkeys. They showed that the recorded signals were different for each object during a reaching movement as well as during a grasp. Somerlik et al. (2008) were able to distinguish three different grip types (cylindrical, tripod, and lateral grip) with an accuracy of more than $82 \%$ using forearm, upper arm and shoulder muscular activity recorded with sEMG. Arguing that lower arm musculature is not available in transradial amputees, they concentrated on upper limb and shoulder muscles for their experiments, where they reached an accuracy of $70 \%$.

Our experiments aim at improving accuracy, distinguishing a larger number of objects, and not only separating shape but also size and weight-related attributes of the object. Furthermore, following Castellini et al. (2009), signal loss in transradial amputees is very limited, so that the use of the remaining forearm musculature will still allow for good results compared to healthy subjects. We therefore decided to focus on forearm musculature only.

\section{Methods}

To record sEMG signals during reaching to grasp, eight OttoBock $13 E 200=50$ surface EMG electrodes (Ottobock) were placed on the subject's forearm (see Fig. A.1), gathering data from the following muscles: extensor carpi radialis longus and brevis, extensor digitorum communis, extensor carpi ulnaris, extensor digiti quinti proprius, flexor carpi radialis, pronator teres, palmaris longus, flexor digitorum superficialis and flexor carpi radialis. Of course, the nature of sEMG signals is such that the recorded signals are not directly related to single muscle activity, but always record a signal based on mixed muscle activity. And further artefacts including skin movement over the muscles during arm/hand movement enhance this. The used sEMG electrodes do not require special skin preparation (e.g., the use of contact gel or shaving). The Otto Bock electrodes include amplification, rectification and filtering electronics; amplification was set to ' 6 ' which corresponds to an amplification factor of about 50,000; bandwidth of the electrodes is specified as $90-480 \mathrm{~Hz}$ with a $-40 \mathrm{~dB}$ notch filter at $50 \mathrm{~Hz}$. The analog signal from the electrodes was then digitized by a National Instruments NI-DAQ 6024E PCMCIA card, providing a 12-bit resolution. The digitized signal was then sampled by a Windows XP PC, which of induced jitter in timing (mean: $0.0022 \mathrm{~s}$, median: $0.0020 \mathrm{~s}$, std: 0.0020 , min: $0.0013 \mathrm{~s}$, max: $0.1865 \mathrm{~s}$ ). Therefore linear interpolation has been applied to get approximated values for the needed point of time (see Appendix A). No further filtering happened after the digital conversion of the electrode signal.

For both experiments the actual object was placed on a platform containing four pressure sensors (FSR type-force sensing resistor) to examine the exact moment of finger-object contact. Visual inspection during a pre-test showed, that the sensors react at the slightest finger-object contact. To be sure to only analyze the part of the EMG-signal before finger-object contact, the last 25 data points (that is approximately $50 \mathrm{~ms}$ ) before sensor reaction were ignored. The subject was instructed to relax his/her hand before starting his/her grasping movement from a fixed position. A fifth pressure sensor at the start position recorded the onset of the hand movement.

\subsection{Experiment 1}

Six healthy subjects were asked to grasp one of eight different objects from 16 different locations. Of the subjects, five were right-handed, one was left-handed, two of them were female, four male. All of them were innocent to the aim of the experiment. The objects to be grasped were: two different sized boxes, a beaker, a cup, a plate, a pencil (in up-right position) and two 0.51 bottles (one empty, one filled with blue colored water). One object a time was placed in front of the subject, who was instructed to grasp the object the way he/she prefers. No requirements of finger placement were provided, but to grasp the same object in the same way every time. Position and kind of the object varied every trial in a randomized way. The object positions were defined as eight positions on a circle in front of the subject and one position in the center of the circle, furthermore, each position varied between a lower and a higher level, concluding in 16 positions in total (see Fig. A.2). With respect to Lederman and Wing (2003) who state that the fingers are positioned on the object such that the lines between the fingers meet in the object's center respective center of mass, each object was placed such that the centers of mass of all objects were at the same height at every specific position, to ensure that this factor does not influence the data. Every subject executed a total of 500 trials.

\subsection{Experiment 2}

Results of the above setting in experiment 1 showed an unsatisfactory classification of the different weighing bottles. Subjects stated that they often were surprised by the weight of the bottles, even though they could clearly see the different filling height of the colored water in the bottles, corresponding to the weight of the bottle. A reason for this might be the high number of trials which lead to distraction of the subjects. Thus an additional experiment was accomplished. To reduce the number of trials only one object position was chosen (in front of the subject, $28 \mathrm{~cm}$ from start to object). Electrodes were placed as described above. Objects to be grasped were three $1 \mathrm{l}$ bottles weighing $100 \mathrm{~g}, 700 \mathrm{~g}$, and $1100 \mathrm{~g}$ and two 0.51 bottles weighing $100 \mathrm{~g}$, and $550 \mathrm{~g}$ and one $1.5 \mathrm{l}$ bottle weighing $1100 \mathrm{~g}$ (same diameter as the 11 bottle, but thinner and more elastic material, thus a more careful grip is required; we

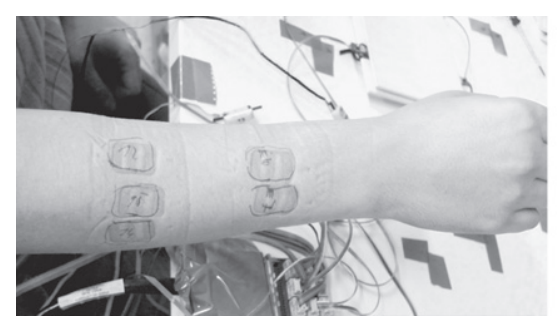

(a) upper side

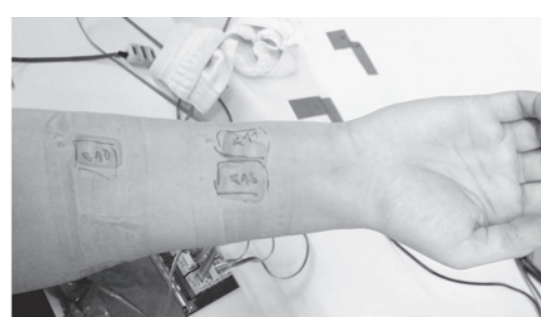

(b) lower side

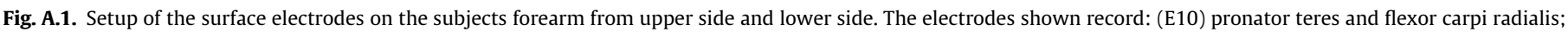

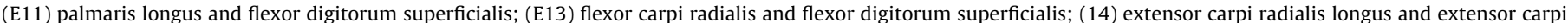

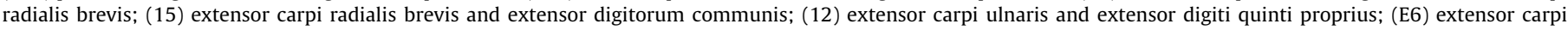
ulnaris and extensor digiti quinti proprius; (E7) extensor digitorum communis and extensor digiti quinti proprius. 


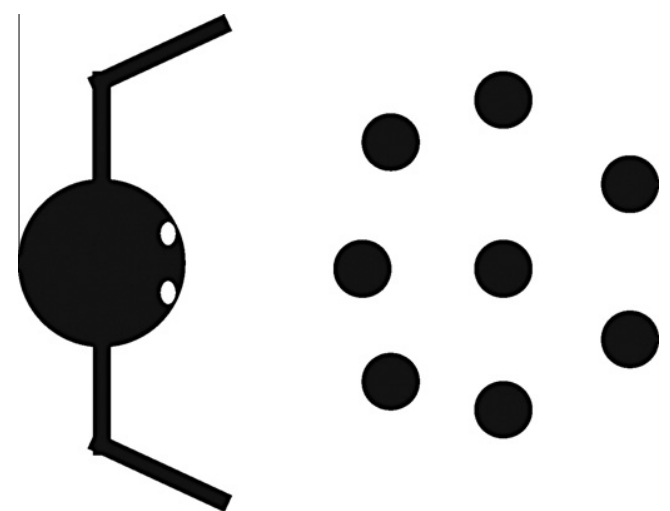

Fig. A.2. Setup of the possible object positions relative to the subject, while the object could be placed on two different heights at each position.

called it 'soft' bottle). As with experiment 1, different weights were achieved by the use of different amounts of colored water filled into the bottles. In contrast to experiment 1 every object had to be grasped five times in a row and only 10 times in total. Finger position at the bottles is defined due to the bottle's geometry, thus for same sized bottles, fingers are placed at the same place independently on the bottle's center of mass. The different sized bottles were placed at such height, that finger position was at same height for every trial. Four right-handed subjects executed this experiment, three of them female and one male. Every subject executed a total of 60 trials.

\subsubsection{Analysis}

Analysis for experiment 1 is carried out for two different settings: one using the signals of all eight electrodes and one using only the signals of the four more proximal electrodes (closer to the elbow). Also three different methods for pre-processing the EMG data are investigated in Appendix A of this paper. Due to the fact, that all of them perform similar accuracy for classification, in the following only method A (see Appendix A) will be used. For classification we use a well-established Support Vector Machine (SVM) implementation called libsvm (Chang and Lin, 2011). Accuracy values are gained by employing 20 -fold cross-validation.

An SVM is a solution to the machine learning problem of binary classification. The basic principle of an SVM is to transform the input data space into a higher dimension an separate two classes via a hyperplane. Since an SVM can only discriminate between two classes, more effort is needed to enable multiple-class classification. libsvm utilizes the 1 -against- 1 method, where each possible pairing of class-combinations is checked, and the class getting most votes is defined as the winning class. For a more in-depth introduction to SVM see, e.g., Burges (1998). A $k$-fold cross-validation, a standard machine-learning approach if only limited data is available, means that the available data is being split into $k$ parts, a SVM is being trained on $k-1$ parts and evaluated on the remaining part. That is repeated to include and test on all of the $k$ parts. So the input into the SVM here is the preprocessed electrode-data according to method A (see Appendix A) and the output is the estimated according class the input data sample belongs to.

The following data is analyzed for each person in experiment 1 :

1. classification of each object against each object using data from all eight EMG electrodes (that is two classes, each containing one object),

2. classification of each object against each object using only data from the four proximal EMG electrodes close to the elbow,

3. classification of six classes using data from all eight EMG electrodes (one class for each object, but full and empty bottle in the same class and beaker and large box in the same class),

4. classification of six classes using only data from the four proximal EMG electrodes close to the elbow (one class for each object, but full and empty bottle in the same class and beaker and large box in the same class),

5. point of time during the reaching movement from which on a reliable classification using data from all eight EMG electrodes can be made (six classes as described above).

Analysis of 1 and 2 is mainly executed to validate the EMG data, while 3 and 4 are the points of interest for application in prosthe-

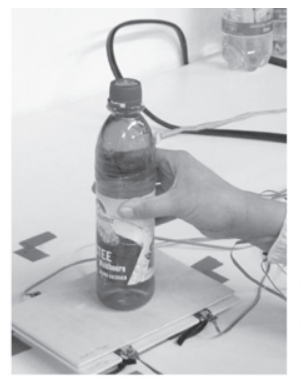

(a) small bottle

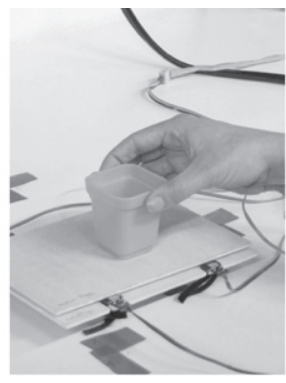

(b) small box

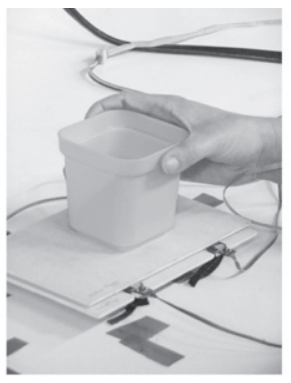

(c) large box

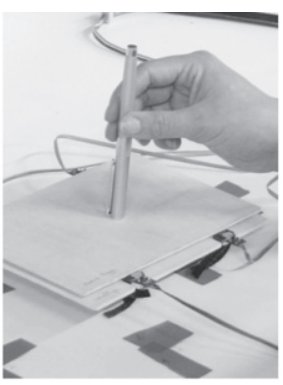

(d) pencil

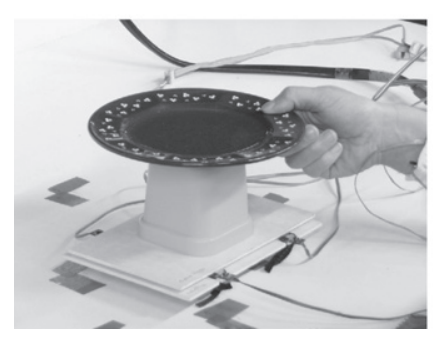

(e) plate

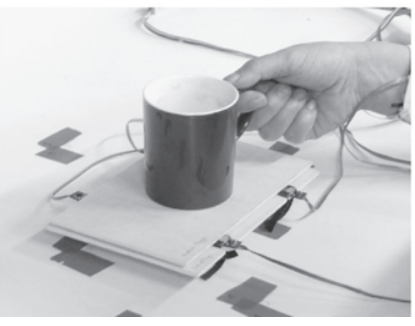

(f) cup

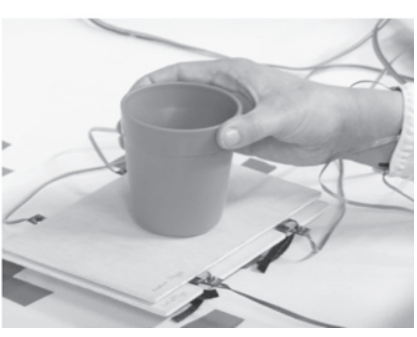

(g) beaker

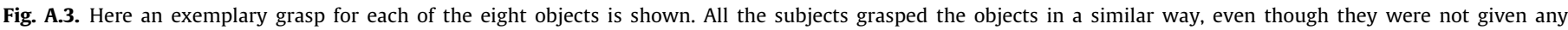
instructions about finger placement. 


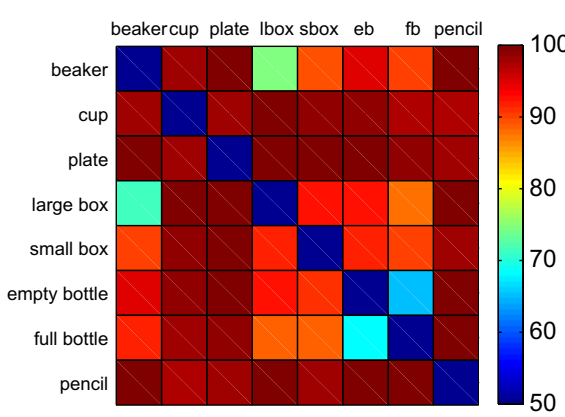

(a) Person 1

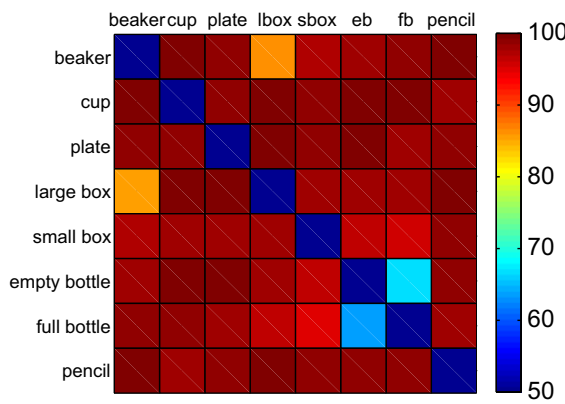

(d) Person 4

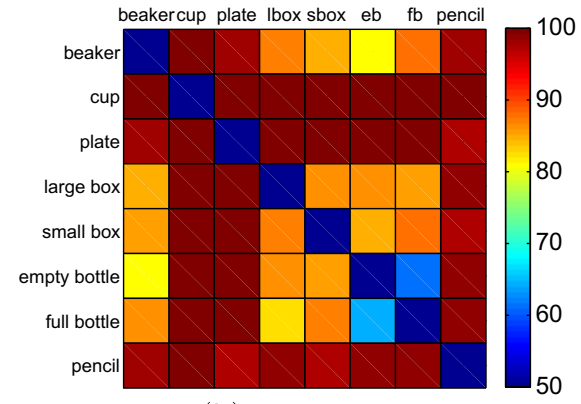

(b) Person 2

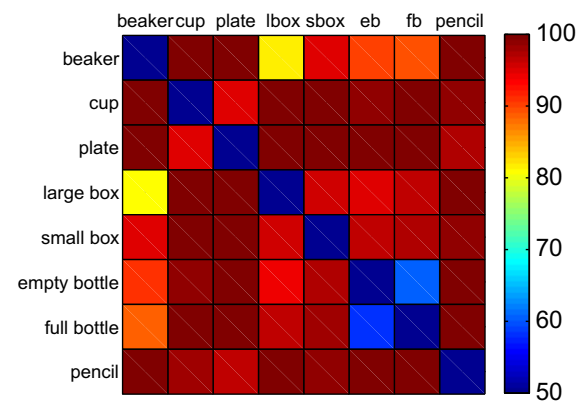

(e) Person 5

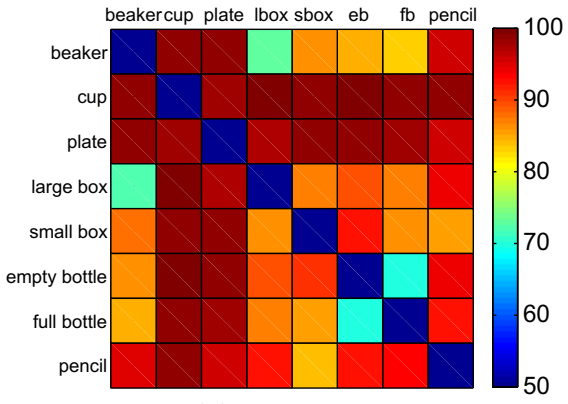

(c) Person 3

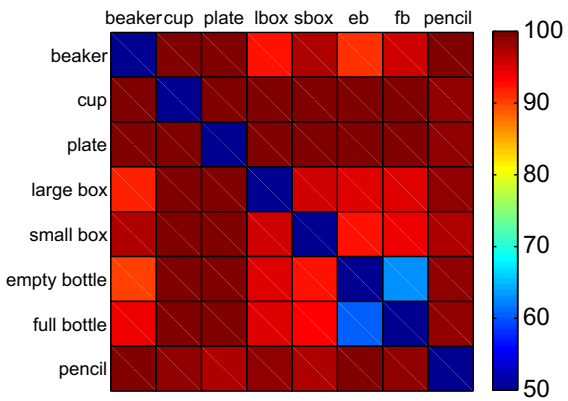

(f) Person 6

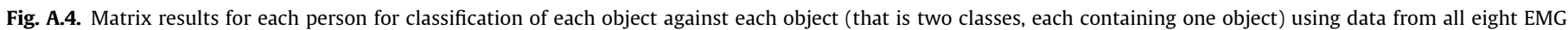
electrodes (near the elbow and near the wrist). Applied method A and 20-fold cross validation.

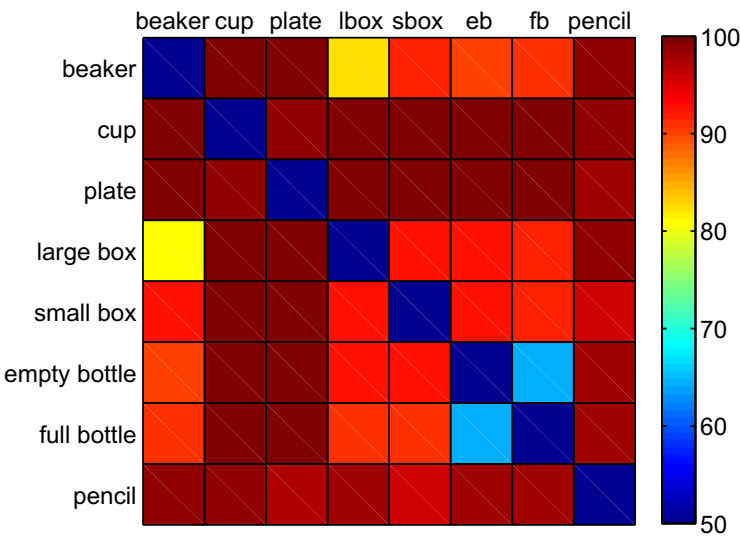

Fig. A.5. Matrix results showing the mean over all persons for using all eight electrodes (near the elbow and near the wrist). Applied method A and 20-fold cross validation.

ses or other artificial hands. The last point is interesting with respect to the level of pre-shaping (Santello and Soechting, 1998; Schettino et al., 2003) as described in the introduction and with respect to the time remaining to (pre-)calculate the movement for the artificial hand.

Analysis of experiment 2 is slightly modified. Accuracy values are gained by employing 10 -fold cross validation:

1. classification of each object against each object using data from all eight sEMG electrodes (that is two classes, each containing one object),

2. classification of six classes using data from all eight sEMG electrodes (one class for each object)

3. point of time during the reaching movement from which on a reliable classification using data from all eight EMG electrodes can be made (using six classes).

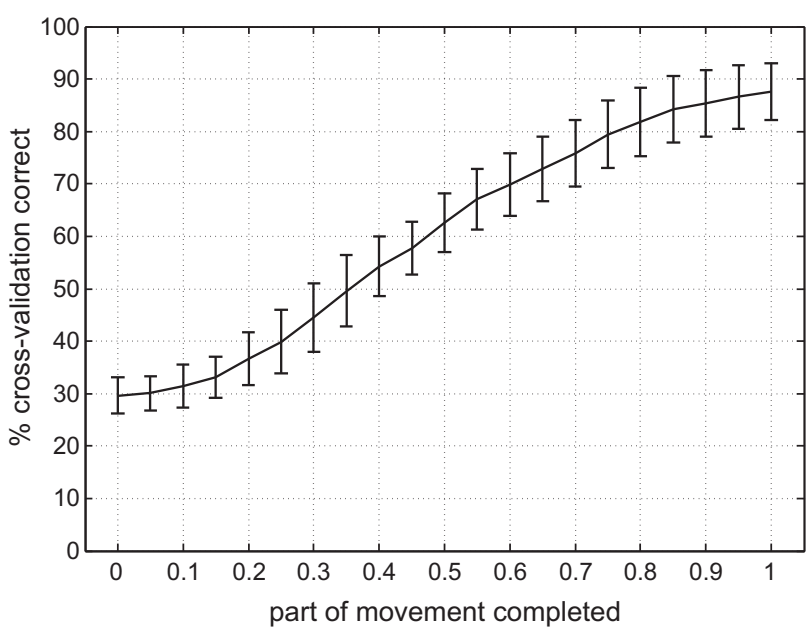

Fig. A.6. This graph visualizes the decrease of classification error the more of the movement is known. Classification has been done on six classes, eight electrodes and deployed method $\mathrm{A}$, showing mean and standard deviation over all persons. Accuracy values gathered by 20 -fold cross validation and pre-processing method A.

\section{Results}

\subsection{Experiment 1}

Even though subjects were not given any instruction regarding the placement of their fingers on the object, all of them grasped the objects in a very similar way. Fig. A.3 shows an exemplary grasp for each object. The empty and the full bottle were grasped the same way. Movement duration was measured as the time between switch release underneath the resting hand, and switch release underneath the object. This duration was measured to vary between $0.02 \mathrm{~s}$ and $2 \mathrm{~s}$ with a median of $0.8 \mathrm{~s}$. Indeed, the measurement method used does not represent very short movements well, but the effect of this inaccuracy has no influence on the out- 


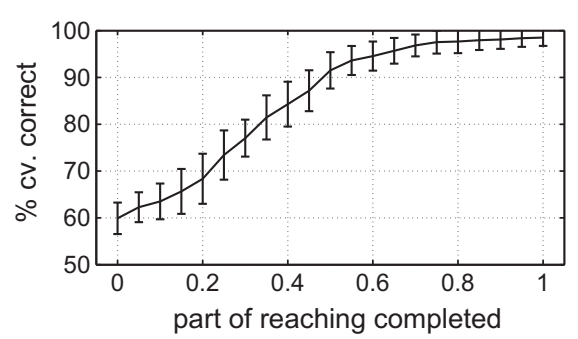

(a) cup and plate

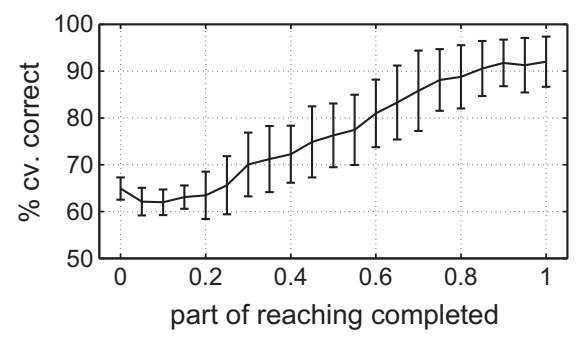

(d) large box and small box

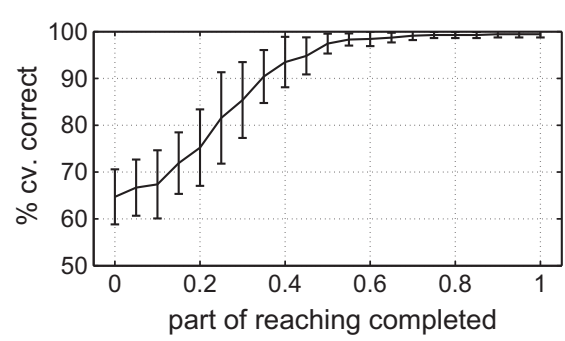

(b) cup and beaker

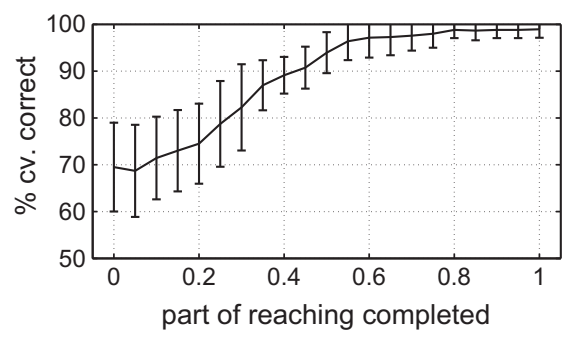

(e) pencil and beaker

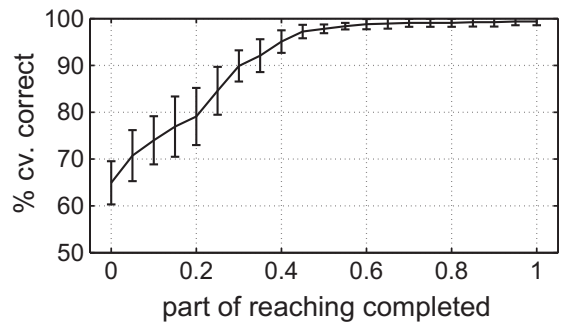

(c) plate and beaker

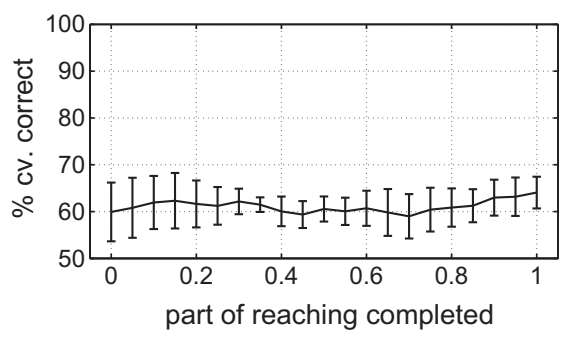

(f) full and empty bottle

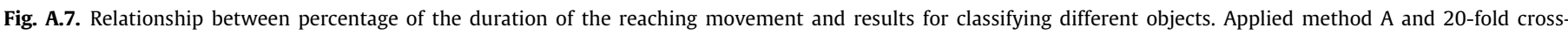
validation.

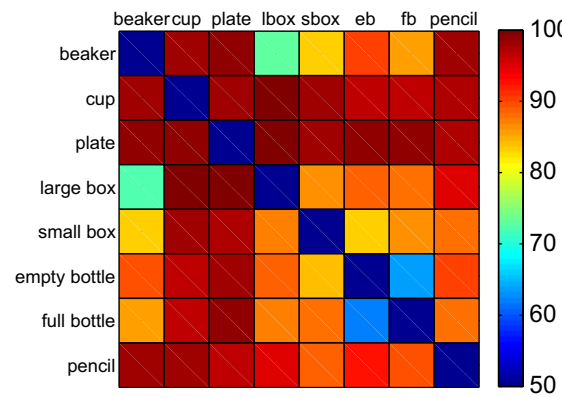

(a) Person 1

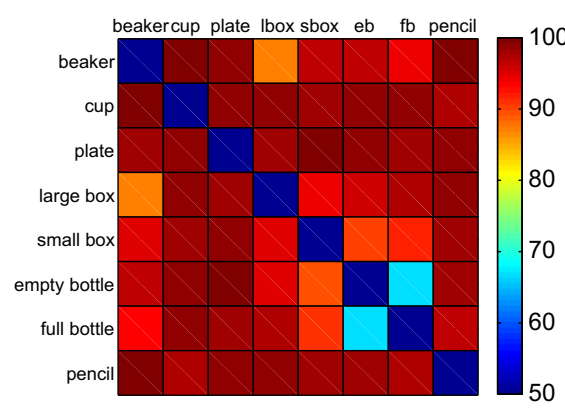

(d) Person 4

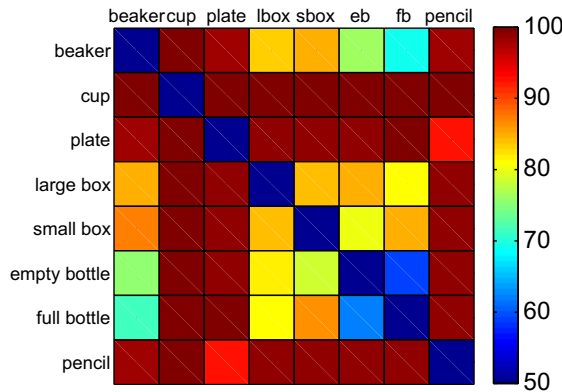

(b) Person 2

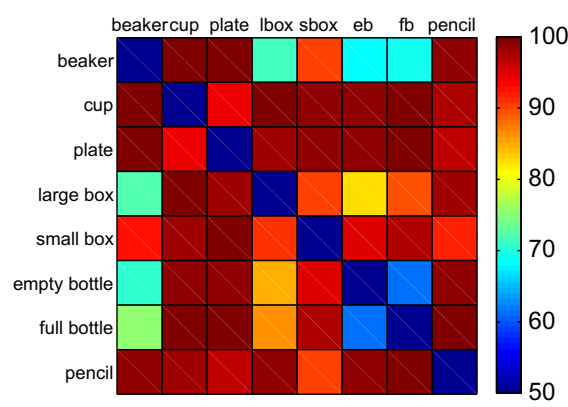

(e) Person 5

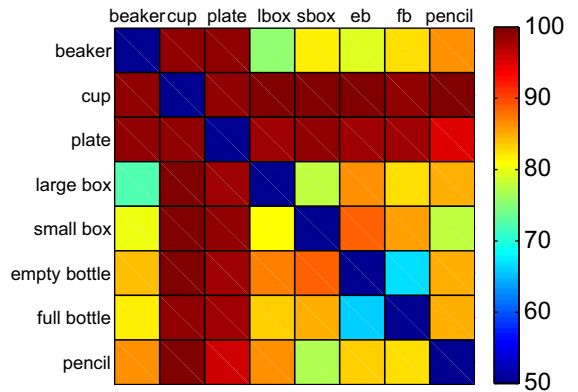

(c) Person 3

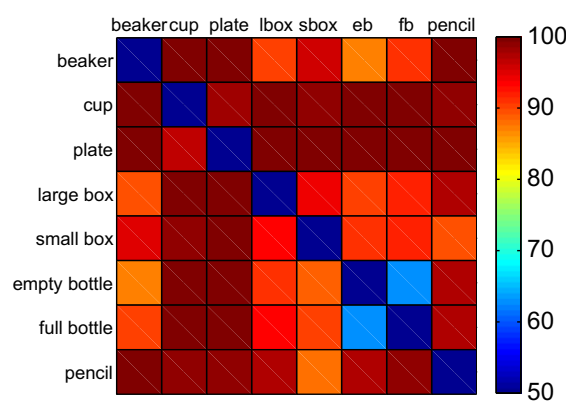

(f) Person 6

Fig. A.8. Matrix results for using only four electrodes near the elbow. Applied method A and 20-fold cross-validation.

come of our experiments. The very short minimal movement durations happen due to the fact that the nearest position has a distance of about $8 \mathrm{~cm}$ from the resting-position detector, so bending the wrist and stretching the fingers allowed to touch the object without releasing the resting-position detector.

\subsubsection{Eight electrodes}

Fig. A.4 shows the results for each person when two objects are classified using method A. Fig. A.5 shows the mean results over all persons. It can be seen, that empty and full bottle can only be distinguished with an accuracy rate of something between $65 \%$ and $75 \%$. That is better than guessing (which would be $50 \%$ ), but in our opinion not sufficient for stable grasping. Thus, for further tests empty and full bottle were put into the same class. For two of the six persons, the distinguishability between large box and beaker is less than $72 \%$. For the other four rises to over $80 \%$. Even though that is much better than for the bottle, we decided to put large box and beaker in the same class for further analysis. The accuracy rate for classifying the remaining six classes lies between $79.1 \%$ and $94.9 \%$ depending on the person, whereas for six classes pure guessing would yield $1 / 6 \approx 17 \%$.

The relationship between part of the reaching movement fulfilled and results for classifying are shown in Fig. A.6. It can be seen that the accuracy rate for six classes at the half of reaching time is about 


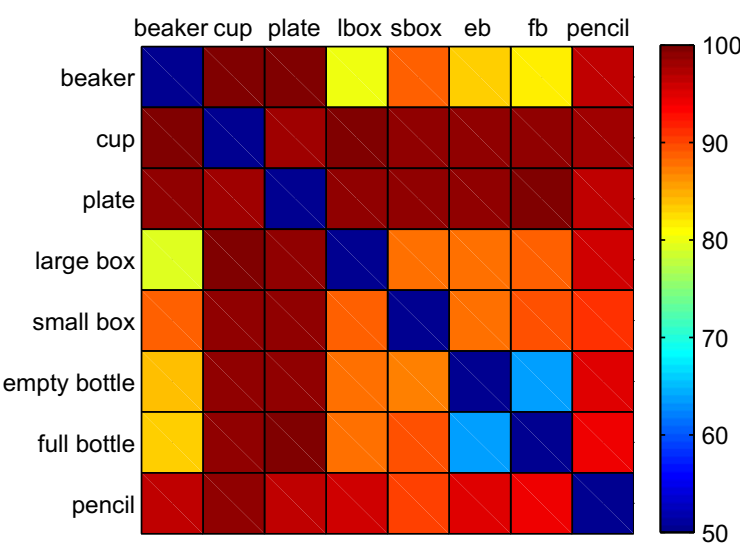

Fig. A.9. Matrix results showing the mean over all persons for using only four electrodes near the elbow. Applied method A and 20-fold cross-validation.

$60 \%$ and improves until the movement is completed. Fig. A.7a-f shows the relationship between part of the reaching movement done and results for classifying two classes. Most of them show a clear distinguishability of the two classes after $50 \%$ of the duration of the reaching. But in Fig. A.7d a significant improvement of the classification can be seen until the movement is completed.

\subsubsection{Four electrodes}

Fig. A.8 shows the results for classifying two objects using only the four electrodes next to the elbow, while Fig. A.9 shows the mean over all participants. The results are not as good as when using all eight electrodes, but still the accuracy is high.

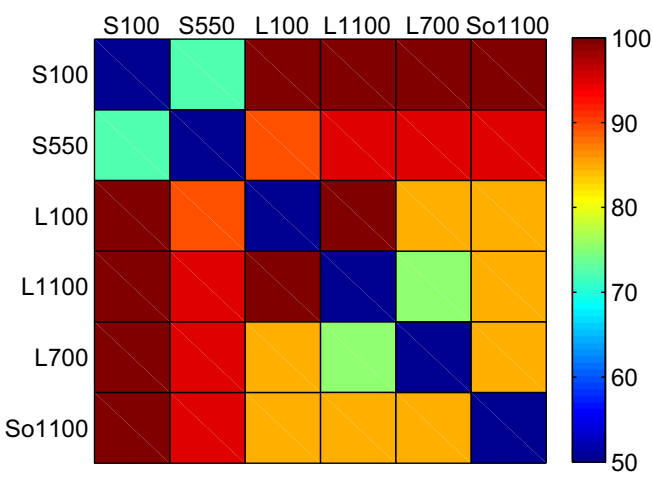

(a) Person 7

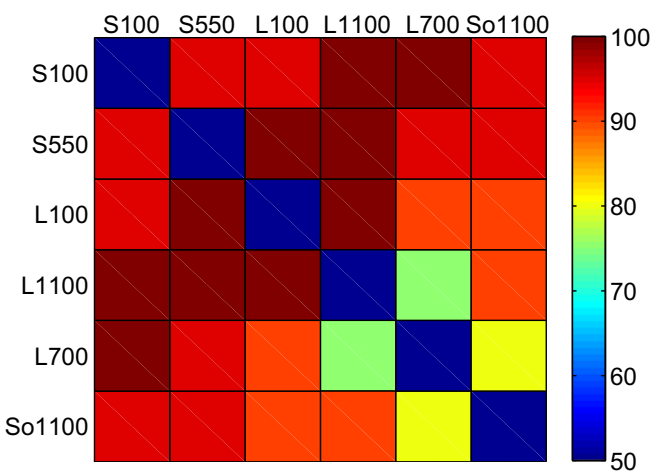

(c) Person 9
For identifying one object out of the six (empty and full bottle considered to be the same class and cup and beaker the same class as described for eight electrodes), accuracy lies between $69.1 \%$ and $90.7 \%$, depending on the person.

\subsection{Experiment 2}

Even though subjects were not given any instruction regarding the placement of their fingers on the bottles, all of them grasped them in a very similar way. The duration of the measured reaching movement varies between $0.43 \mathrm{~s}$ and $1.49 \mathrm{~s}$ with a median of $0.73 \mathrm{~s}$.

Fig. A.10 shows the results when always two different objects are classified against each other using method A. Accuracy rates of the 0.51 bottles range from $73 \%$ to $94 \%$ and are thus better than in experiment 1 (where they ranged from 65\% to 75\%), still differing considerably between participants.

As already seen in experiment 1 (see Figs. A.7f and A.12), within the case of different object weight and similar object size and shape, accuracy rate does not improve very much over time, but stays almost the same from the beginning on (see Fig. A.11).

\section{Discussion}

Matrix results for classifying two objects (Figs. A.4, A.8 and A.10) show that there are inter-person differences. Especially persons 4-6 of experiment 1 show very good results. The inter-person differences may depend on different anatomical properties of the subjects like muscle strength, skin impedance, amount of fatty tissue and many more as well as on a feasible grasping repeatability of each person.

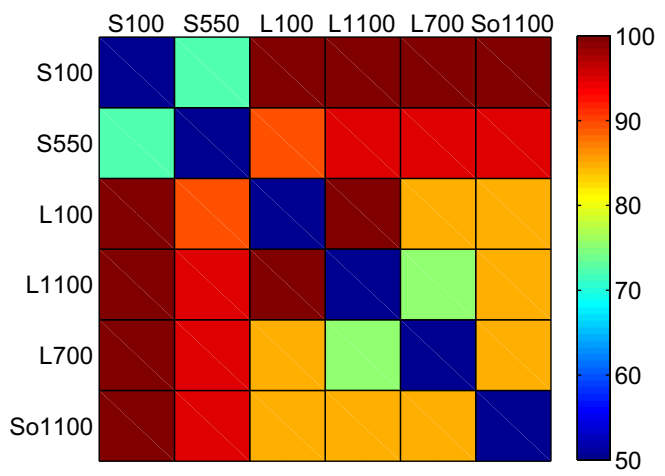

(b) Person 8

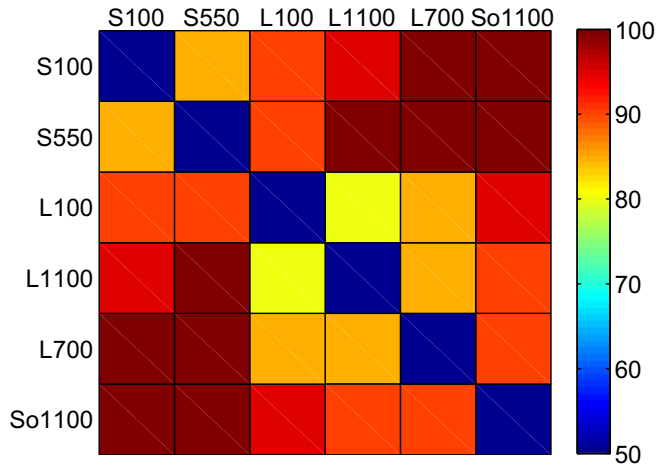

(d) Person 10

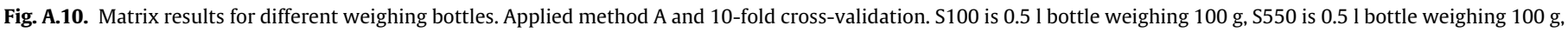
L100 is $1 \mathrm{l}$ bottle weighing $100 \mathrm{~g}$, L700 is $1 \mathrm{l}$ bottle weighing $700 \mathrm{~g}$, L1100 is $1 \mathrm{l}$ bottle weighing $1100 \mathrm{~g}$, So1100 is $1.5 \mathrm{l}$ bottle ('soft' bottle) weighing $1100 \mathrm{~g}$. 


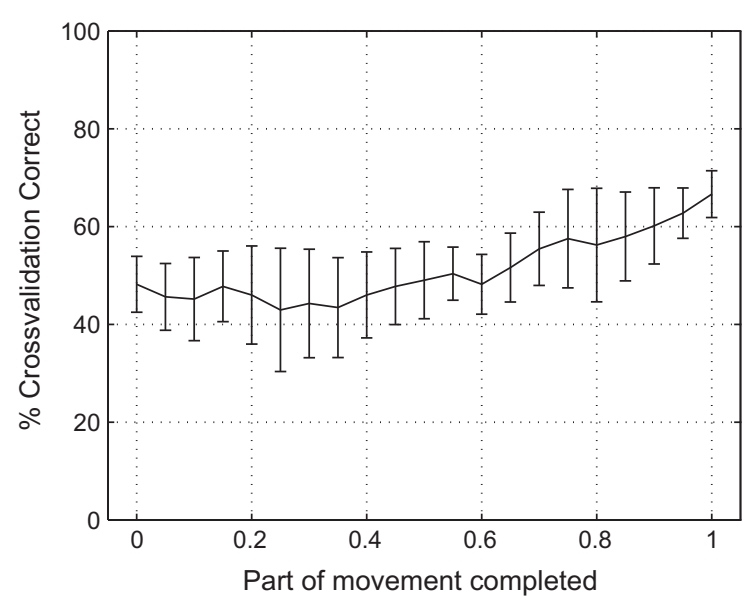

Fig. A.11. This graph visualizes the decrease of classification error the more of the movement is known. Classification has been done on six classes, eight electrodes and deployed method A, showing mean and standard deviation over all persons. Accuracy values gathered by 10 -fold cross validation.

The relationship between fulfilled part of the reaching movement and results for classifying two classes (see Fig. A.7a-f) lead to the conclusion, that objects shape may be distinguished in the first half of the movement, but distinguishability of object size improves over the whole reaching movement.

This leads to the conclusion, that not only maximum aperture (which depends on object size as described in Jeannerod (1981) and Paulignan and Jeannerod (1996) and is supposed to happen midway of the reaching movement), but also hand posture (which develops during the whole reaching movement (Santello and Soechting, 1998)) are dependent on object properties and are visible in the EMG signal. On the other hand, Schettino et al. (2003) describe that hand postures requiring only configuration changes in abduction/adduction dimension occur at about $45 \%$ of the reaching movement (in contrast to changes in the flexion/extension dimension which occur only after $75 \%$ of the movement).

Further reasons for the ongoing improvement of classification accuracy may be that the distances from the starting position to the object varied a lot in our setup, so-especially for the short distances-the movement duration may in some cases be too short. Another aspect is the signal to noise ratio of the electrodes, thus it is natural, that the better the data gets, the better the classification result will be. Therefore a monotonic raising error function up to the end of the movement is quite normal, however we would have awaited to see a steeper slope up to the 50\% mark. Furthermore we presume that persons may already have started their pre-shaping or in some sense pre-stiffening their muscles before they lifted the hand from the pressure sensor detecting the onset of the movement. This might explain, why Fig. A.6 starts at about $30 \%$, where pure chance for guessing within six classes would be about $17 \%$.

In this context, a mentionable aspect is shown in Fig. A.7f, showing the classification over duration of the two bottles with different weight in experiment 1 . The accuracy rate is not improving over time but is fixed around $60 \%$ from the beginning (while pure guessing would be 50\%). We may conclude that the weight leads to an early decision of pre-shaping, namely setting the grasp impedance to cope with the expected object weight. Fig. A.12 from experiment 2 reinforces this finding. Close inspection of those figures, however, reveal that the larger the difference in weight gets, the more the development of decision accuracy takes place over the whole time, but not as dramatically as seen on pre-shaping depending on shape and size.

The overall results lead to the conclusion that it is possible to recognize different object sizes (like small and large box) and grasp types (like power grasps for boxes, bottles and beaker, fine grasp for the pencil or special grasps for the plate and the cup) with sEMG signals during the reaching phase well before the grasp is actually performed.

Results of experiment 1 for persons 4 and 6 using only the four proximal electrodes show that accuracy rates can be above $90 \%$ for this setting as well. And this setting is feasible for a wide range of amputees (approximately trans-radial up to one-thirds proximal; despite the setting with eight electrodes, which is only feasible for amputees trans-radial up to one third distal). These results compare favorably to those reported by Somerlik et al. (2008). There, an accuracy rate of $82.3 \%$ in three classes using forearm, upper arm, and shoulder muscles was obtained when classifying over the full movement. In our experiments, the same accuracy is obtained for six classes after $80 \%$ of the whole reach movement.

Even though the results for classifying object weight (empty and full bottle) were not as good as for classifying object size or grasp type, it can be seen that there is information about the object weight in the sEMG signal before contact. Furthermore it is not clear if the person really concentrated on object weight or (pre)estimated a wrong weight, since this was not checked within/after the trial. However feedback after experiment 1 showed that the test persons found it hard to remain concentrated throughout the 500 grasps, and especially the different bottles surprised them, even though the liquid was colored blue to give a visual cue. For this reason we conducted experiment 2 with only a

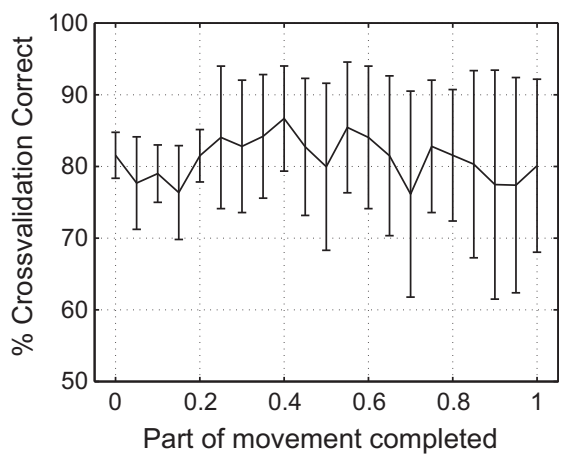

(a) small b. $90 \mathrm{~g}$ and $560 \mathrm{~g}$

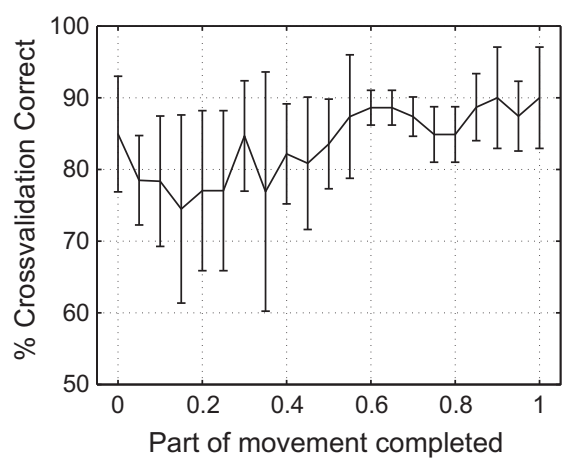

(b) large b. $90 \mathrm{~g}$ and $700 \mathrm{~g}$

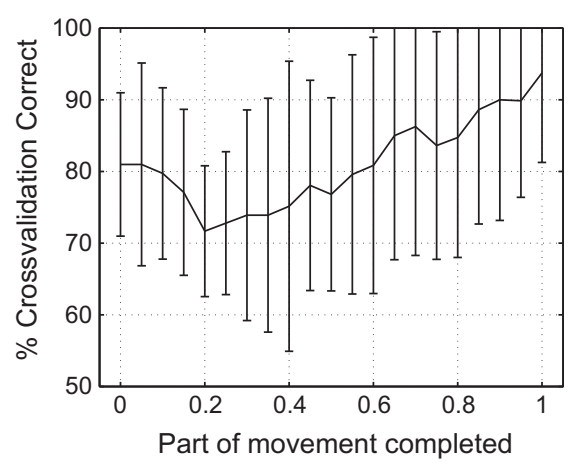

(c) large b. $90 \mathrm{~g}$ and $1100 \mathrm{~g}$

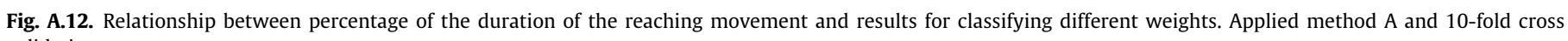
validation. 
limited number of grasps to be executed. Results are better than for experiment 1 , but show, that a large difference in weight is necessary to receive accuracy rates above $90 \%$. A mentionable detail in the results of experiment 2 is that the soft bottle can be easily distinguished from the large bottle, even so they have the same diameter and weight. Summing up the thoughts on different weights of the objects, it seems, that shape and size as well as surface properties (like 'soft bottle') have more impact on the muscular system during pre-shaping than the actual weight of the to be grasped object. This is quite surprising, since different work (e.g., Hermsdörfer et al., 1999) showed that force is always modulated such that the least possible amount necessary for a stable grasp is used, even in dynamic scenarios.

This work clearly shows that some useful information of the to be grasped object is available well before contact-from about 50$70 \%$ of total movement time. How to extract and process this information while grasping an object with prosthesis is however not within the scope of this work, but will extend into further research.

\section{Acknowledgments}

This work has been partially funded by the European Commission's Seventh Framework Programme as part of the Project STIFF under Grant No. 231576 and THE under Grant No. 248587.

\section{Appendix A}

Three different methods are used to pre-process the EMG signals to generate a feature vector $\mathbf{x}$ from each trial consisting of 15 equidistant sampling points $\mathbf{s}_{t_{i}} ; i \in\{1,2, \ldots, 15\}$, with each $\mathbf{s}_{t_{i}}$ being the signal from the 8 (4) sEMG electrodes at time $t_{i}$.

For pre-processing of the data we investigated three different approaches.

\section{A.1. Method A}

Signals are normalized over time. This is necessary since the durations of the movements are highly variable, some last as short as about $0.02 \mathrm{~s}$ and some last up to about $2 \mathrm{~s}$. So rather of taking time-equidistant samples, we decided to take them equally distributed along the movement. Let $t_{1}$ be the time when the movement starts (i.e., the subject lifts the hand), and $t_{n}$ the end of movement (i.e., the subject touches the object). The equally distributed points of time $t_{i}$ are then defined as:

$t_{i}=(i-1) \frac{t_{n}-t_{1}}{n-1}$,

with $n$ as the total number of equidistant sampling points. Subsequently the EMG-data at each sampling time is concatenated into a single feature vector $\mathbf{x}^{A}$ :

$\mathbf{x}^{A}=\left[\mathbf{s}_{t_{1}}^{T}, \mathbf{s}_{t_{2}}^{T}, \ldots, \mathbf{s}_{t_{n}}^{T}\right]^{T}$

with $n=15$.

\section{A.2. Method B}

The integral over each EMG signal is calculated and used as an extra input added to method A, resulting in $16(8)$ instead of 8 (4) inputs. The idea behind this pre-processing is to give the classifier also some kind of energy-measure of the movement; a slow movement results into a lower amplitude, whereas a fast movement has a higher amplitude. The pre-processing in Method A however discards the information regarding the real length of the movement, yet leaves the amplitude data in. Therefore the integral re-adds this missing information. To scale the value of the integral signals
Table 1

Results for classification via 20 -fold cross-validation of six classes using different preprocessing methods using data collected from experiment 1.

\begin{tabular}{llll}
\hline Subject & Method A & Method B & Method C \\
\hline Person 1 & $86.5 \%$ & $86.3 \%$ & $86.7 \%$ \\
Person 2 & $84.4 \%$ & $84.4 \%$ & $83.2 \%$ \\
Person 3 & $79.7 \%$ & $79.7 \%$ & $78.3 \%$ \\
Person 4 & $94.9 \%$ & $95.5 \%$ & $94.7 \%$ \\
Person 5 & $90.1 \%$ & $90.3 \%$ & $89.1 \%$ \\
Person 6 & $90.2 \%$ & $90.2 \%$ & $90.6 \%$ \\
Mean & $87.7 \%$ & $87.8 \%$ & $87.1 \%$ \\
Deviation & 5.30 & 5.50 & 5.78 \\
\hline
\end{tabular}

back into the magnitude of the sEMG electrodes values, the integral-values are all scaled down by the mean of each integrated signals maximum value together with a mixing factor ${ }^{1}$ of $1 / 2$.

We define

$d\left(\mathbf{s}_{t_{i}}\right)=\sum_{k=0}^{t_{i}} \mathbf{s}_{k}$

while the sum is evaluated per element so that $d\left(\mathbf{s}_{t_{i}}\right)$ is a vector of the same dimension as $\mathbf{s}_{t_{i}}$. Taking mean(a) as the mean of all elements of the vector $\mathbf{a}$, we set

$p\left(\mathbf{s}_{t_{i}}\right)=\frac{1}{2} \frac{d\left(\mathbf{s}_{t_{i}}\right)}{\operatorname{mean}\left(d\left(\mathbf{s}_{t_{n}}\right)\right)}$,

while, again, $p\left(\mathbf{s}_{t_{i}}\right)$ is a vector of the same dimension as $\mathbf{s}_{t_{i}}$ and $n=15$. Effectively $p\left(\mathbf{s}_{t_{i}}\right)$ is the normalized vector of summed EMG signal up to $t_{i}$. The resulting feature vector $\mathbf{x}^{B}$ is

$\mathbf{x}^{B}=\left[\mathbf{s}_{t_{1}}^{T}, \mathbf{s}_{t_{2}}^{T}, \ldots, \mathbf{s}_{t_{n}}^{T}, p\left(\mathbf{s}_{t_{1}}\right)^{T}, p\left(\mathbf{s}_{t_{2}}\right)^{T}, \ldots, p\left(\mathbf{s}_{t_{n}}\right)^{T}\right]^{T}$,

with $n=15$.

\section{A.3. Method C}

Each sEMG signal is divided by the maximum sEMG value for the specific trial. This has to be seen as some kind of normalization, so that all trials have the same amplitude. This is an ambivalent kind of pre-processing. On the one hand, it ensures, that all $\mathbf{x}$ are of the same amplitude-slow, low amplitude movements get the same as fast, high amplitude movements-but on the other hand, some information might also be encoded in the maximum amplitude, and we will loose this, of course. The normalization factor $\alpha$ as:

$\alpha=\max \left(\max \left(\mathbf{s}_{t_{1}}\right), \max \left(\mathbf{s}_{t_{2}}\right), \ldots, \max \left(\mathbf{s}_{t_{n}}\right)\right)$,

assuming max (a) gives the maximal element of the vector a. The feature vector $\mathbf{x}^{C}$ is then constructed analogous to method $A$

$\mathbf{x}^{C}=\frac{1}{\alpha}\left[\mathbf{s}_{t_{1}}^{T}, \mathbf{s}_{t_{2}}^{T}, \ldots, \mathbf{s}_{t_{n}}^{T}\right]^{T}$

with $n=15$.

\section{A.4. Results}

There are only small differences in the classifications results between methods A, B and C. Normalization method B performs slightly better results than A and C, however the standard deviation clearly shows that this increase in accuracy is far below

\footnotetext{
${ }^{1}$ We conducted a series with multiple mixing factors, where $1 / 2$ yielded the best results, but since the impact of the different pre-processing methods is so marginal,
} we omit this for brevity. 
inter-person variances. See Table 1 for details. Since the differences are so marginal, we used method A for the sake of simplicity.

The classification of $\mathbf{X}=\left\{\mathbf{x}_{1}, \ldots, \mathbf{x}_{k}\right\}$, with $k$ as the total number of training samples, is being done by a very well established Support Vector Machine (SVM) implementation called libsvm (Chang and Lin, 2011). For accuracy values we used 20 -fold cross-validation.

\section{References}

Bitzer S, van der Smagt P. Learning EMG control of a robotic hand: towards active prostheses. In: Proceedings of the IEEE international conference on robotics and automation; 2006. p. 2819-23.

Brochier T, Spinks R, Umilta M, Lemon R. Patterns of muscle activity underlying object specific grasp by the macaque monkey. J Neurophysiol 2004;92:1770-82

Burges CJC. A tutorial on support vector machines for pattern recognition. Data Min Knowl Discovery 1998;2:121-67.

Castellini C, Gruppioni E, Davalli A, Sandini G. Fine detection of grasp force and posture of amputees via surface electromyography. J Physiol Paris 2009;103(35):255-62.

Castellini C, van der Smagt P. Surface EMG in advanced hand prosthetics. Biol Cyber 2009;100(1):35-47.

Chang CC, Lin CJ. LIBSVM: a library for support vector machines. ACM Trans Intell Syst Technol 2011;2:27:1-27:27. <http://www.csie.ntu.edu.tw/cjlin/libsvm>

Cutkosky M, Howe R. Human grasp choice and robotic grasp analysis. SpringerVerlag New York, Inc.; 1990. p. 5-31.

Eastough D, Edwards M. Movement kinematics in prehension are affected by grasping objects of different mass. Exp Brain Res 2007;176:193-8.

Hermsdörfer J, Marquardt C, Philipp J, Zierdt A, Nowak D, Glasauer S, et al. Grip forces exerted against stationary held objects during gravity changes. Exp Brain Res 1999;126:205-14.

Jeannerod M. Intersegmental coordination during reaching at natural visual objects. Erlbaum; 1981. p. 153-68.

Lederman S, Wing A. Perceptual judgement, grasp point selection and object symmetry. Exp Brain Res 2003;152:156-65.

Ottobock. <http://www.ottobock.de>.

Parker P, Englehart K, Hudgins B. Myoelectric signal processing for control of powered limb prostheses. J Electromyogr Kinesiol 2006;6:541-8.

Paulignan Y, Jeannerod M. Prehension movements. The visuomotor channels hypothesis revisited. CA (USA): Academic Press; 1996. p. 265-82.

Santello M, Soechting J. Gradual molding of the hand to object contours. J Neurophysiol 1998;79(3):1307-20.

Schettino L, Adamovich S, Poinzer H. Effects of object shape on hand configuration during grasping. Exp Brain Res 2003;151(2):158-66.

Somerlik K, Krueger T, Carpaneto J, Stieglitz T, Micera S. Physiologic approach for control of hand prostheses. In: IFMBE proceedings 22; 2008. p. 1830-4.

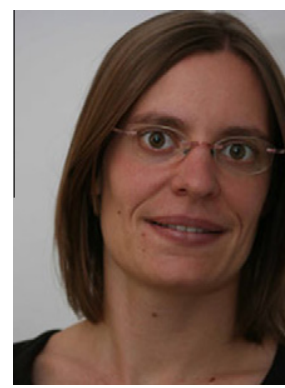

Nadine Fligge studied electrical engineering at the Technical University in Munich until 2007. Since then she has been working as a research engineer at the Institute of Robotics and Mechatronics at the German Aerospace Center (DLR). Her main interests are investigation and modeling of human behavior, especially grasping.

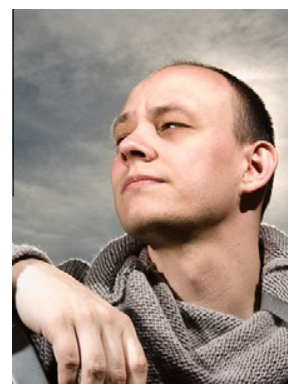

Holger Urbanek studied computer science at the Technische Universität München, Germany, and finished with a diploma in 2003. Since then he has been a research associate at the Institute of Robotics and Mechatronics at the German Aerospace Center (DLR). His research interests include machine learning, inverse problems and surface electromyography.

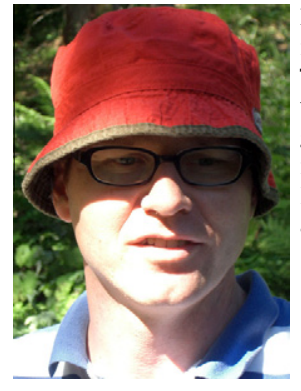

Patrick van der Smagt Leading a lab on biomimetic robotics at the DLR and a machine learning lab at the Technische Universität München, he is author of a whole bunch of papers to which he contributed to greater or lesser extent. He is increasingly aware of the importance of open access publishing, and tries to push his lab to understanding these publication issues for the advancement of science. 